\title{
ASYMPTOTIC ANALYSIS OF A ROD WITH SMALL BENDING STIFFNESS
}

\author{
BY \\ PETER WOLFE \\ University of Maryland, College Park, Maryland
}

\begin{abstract}
In this paper we consider a model problem for the deformation of a rod with small bending stiffness. We show that this problem can be considered as a singular perturbation of the problem in which the rod is replaced by a string with no resistance to bending. We construct an approximate solution to this problem. As the bending stiffness tends to zero this solution tends to the solution of the string problem away from the ends of the rod which are assumed to be clamped. However, as one would expect, there is a boundary layer near each end of the rod. The main point of the paper is to show how to construct the boundary layer corrections.
\end{abstract}

0. Introduction. The object of this paper is to begin to explore a theory of the deformation of a rod with small bending stiffness. The simplest model of one-dimensional elasticity is the string. A string is assumed to be perfectly flexible, i.e., it offers no resistance to bending. Of course the notion of a perfectly flexible string is an idealization. Actual strings or wires do offer at least some resistance to bending and thus any realistic model must take this into account. The model we use here is that of the Cosserat rod. (We use the term rod to denote a slender three-dimensional body.) Here we assume the rod may undergo extension and flexure but not shear. The problem we wish to study is that of a conducting wire in a magnetic field. The magnetic field is constant in magnitude and perpendicular to the line between the supports of the wire. This gives rise to a planar problem. When we model the wire as a string, the problem can be solved explicitly. When we model the wire as a rod the equations naturally become more complicated since we must include the effects of bending moments. Also, in addition to stipulating the positions of the ends of the wire as we do in the string problem, we must impose additional boundary conditions. In our case we impose the condition that the wire is clamped at both ends. We consider a continuum of rod problems depending on a parameter $\varepsilon$. As $\varepsilon$ tends to zero the wire becomes more and more "stringlike" in the sense that the bending stiffness tends to zero. Mathematically, then, we consider the rod model as a singular perturbation of the string problem. Thus we would expect that as $\varepsilon$ tends to zero the solution of the rod problem tends to the solution of the rod problem in the interior of the wire. However near the ends of the wire there must be a boundary layer. This means that

Received December 27, 1989.

(C)1991 Brown University 
for small $\varepsilon$ some components of the solution must undergo large variations near the ends of the wire in order to satisfy the additional boundary conditions. In this paper we show how to construct an asymptotic solution to the rod problem which tends to the solution of the string problem as $\varepsilon$ tends to zero. The main point of this paper is to show how to construct the boundary layer corrections. For this we make an ansatz for the form of the solution. We then derive and solve the resulting boundary layer equations. In making the ansatz we were guided by the work of Flaherty and O'Malley [2].

The physical problem we wish to consider is this. We have a nonlinearly elastic conducting wire whose two ends are fixed. The wire carries a current $I$. There is a constant magnetic field whose direction is perpendicular to the line between the ends of the wire. The force that results from the interaction of the current and the magnetic field produces a deformation in the wire which will take place in the plane perpendicular to the direction of the magnetic field. In Sect. 1 we will describe the rod model and our concept of small bending moments. In Sect. 2 we will describe the string model and the solution to the string problem. In Sect. 3 we will construct the asymptotic solution to the rod problem.

1. The rod model. In this section we model the wire as a rod. By a rod we understand a slender three-dimensional body. Let $\{\mathbf{i}, \mathbf{j}, \mathbf{k}\}$ be a fixed orthonormal basis in Euclidian 3-space, $E^{3}$. We define a position vector function $\mathbf{r} \in E^{3}$ of the real variable $s \in[0,1]$. Here $s$ is interpreted as a scaled arc length parameter of the line of centroids of the rod in a reference configuration so $s$ identifies material cross sections of the rod. Thus in a deformed configuration $\mathbf{r}(\mathbf{s})$ is the position of the material point at the centroid of the section $s$. The wire (rod) is assumed to be welded to fixed supports located at $\pm b / 2 \mathbf{i}$ where $b>1$. The wire caries a current $I$. We suppose that there is a magnetic field $\mathbf{B}=B \mathbf{k}$ present. The force on the wire is then given by

$$
\mathbf{f}(\mathbf{s})=I \mathbf{r}^{\prime} \times \mathbf{B} .
$$

Here and elsewhere prime denotes differentiation with respect to $s$. Let $\mathbf{n}(\mathbf{s})$ denote the contact force and $\mathbf{m}(\mathbf{s})$ denote the contact couple or bending moment exerted by the material of $(s, 1]$ on the material of $[0, s]$.

Then the equations of equilibrium for the rod are

$$
\begin{gathered}
\mathbf{n}^{\prime}+\mathbf{f}=\mathbf{0}, \\
\mathbf{m}^{\prime}+\mathbf{r}^{\prime} \times \mathbf{n}=\mathbf{0} .
\end{gathered}
$$

We look for solutions of the problem for which $\mathbf{r}(\mathbf{s})$ lies in span $\{\mathbf{i}, \mathbf{j}\}$, i.e., we consider planar deformations of the rod so that $\mathbf{r}=x \mathbf{i}+y \mathbf{j}$. Thus the equilibrium equations become

$$
\begin{aligned}
& \mathbf{n}^{\prime}+\lambda\left(\mathbf{y}^{\prime} \mathbf{i}-\mathbf{x}^{\prime} \mathbf{j}\right)=\mathbf{0} \\
& M^{\prime}+\left(\mathbf{r}^{\prime} \times \mathbf{n}\right) \cdot \mathbf{k}=\mathbf{0},
\end{aligned}
$$

where

$$
\lambda=I B
$$


and

$$
M=\mathbf{m} \cdot \mathbf{k} .
$$

We assume here that $\lambda>0$. The sign of $\lambda$ is inessential. We further assume that the rod is unshearable so that the material section of the rod at $s$ remains perpendicular to $\mathbf{r}^{\prime}(\mathbf{s})$ in the deformed configuration. We now set

$$
\begin{gathered}
x^{\prime}=v \cos \theta, \quad y^{\prime}=v \sin \theta, \\
\mathbf{e}_{1}=\cos \theta \mathbf{i}+\sin \theta \mathbf{j}, \\
\mathbf{e}_{2}=-\sin \theta \mathbf{i}+\cos \theta \mathbf{j},
\end{gathered}
$$

so that

$$
\mathbf{r}^{\prime}=v \mathbf{e}_{1}
$$

We decompose $\mathbf{n}$ as

$$
\mathbf{n}=N \mathbf{e}_{1}+h \mathbf{e}_{2}
$$

and set

$$
\theta^{\prime}=u \text {. }
$$

Then $u$ and $v$ are the strains of the problem. We take the dot product of (1.4) with $\mathbf{e}_{1}$ and $\mathbf{e}_{2}$ and use (1.8), (1.9), and (1.10). We then find that the system (1.4), (1.5) becomes

$$
\begin{gathered}
N^{\prime}-u h=0, \\
h^{\prime}+u N-\lambda v=0, \\
M^{\prime}+v h=0 .
\end{gathered}
$$

The elastic properties of the rod are embodied in the constituative equations relating the stresses $M$ and $N$ to the strains $u$ and $v$. Thus we assume that there are continuously differentiable functions

$$
\mathbb{R} \times(0, \infty) \ni(u, v) \rightarrow(\widehat{M}(u, v), \widehat{N}(u, v)) \in \mathbb{R}^{2}
$$

such that

$$
M(s)=\widehat{M}(u(s), v(s)), \quad N(s)=\widehat{N}(u(s), v(s)) .
$$

The ratio $M / u$ which is constant in the linear theory will be referred to as the bending stiffness. We further assume that the rod is hyperelastic, meaning that there is a stored energy function $\Phi(u, v)$ such that

$$
\widehat{M}(u, v)=\Phi_{u}(u, v), \quad \widehat{N}(u, v)=\Phi_{v}(u, v), \quad \text { for }(u, v) \in \mathbb{R} \times(0, \infty) .
$$

Here subscripts denote partial derivatives. We assume $\Phi$ is twice continuously differentiable, convex, and coercive in the sense that

$$
\begin{gathered}
\Phi(u, v) /\left(u^{2}+v^{2}\right)^{\alpha} \rightarrow \infty \text { as } u^{2}+v^{2} \rightarrow \infty \text { for some } \alpha>1, \\
\Phi(u, v) \rightarrow \infty \text { as } v \rightarrow 0 \\
{\left[\begin{array}{cc}
\widehat{M}_{u} & \widehat{M}_{v} \\
\widehat{N}_{u} & \widehat{N}_{v}
\end{array}\right] \quad \text { is positive definite. }}
\end{gathered}
$$


Equations $(1.14 \mathrm{a}, \mathrm{b})$ imply

$$
\begin{aligned}
& \widehat{N}(u, v) \rightarrow \begin{cases}-\infty & \text { as } v \rightarrow 0, \\
\infty & \text { as } v \rightarrow \infty\end{cases} \\
& \widehat{M}(u, v) \rightarrow \pm \infty \text { as } u \rightarrow \pm \infty
\end{aligned}
$$

Finally we assume

$$
\widehat{N}(0,1)=0, \quad \widehat{M}(0, v)=0 .
$$

See [1] for a discussion of the physical significance of these conditions. In this model the stress $h$ is an unknown of the problem. The boundary conditions reflecting the condition that the rod is clamped at both ends are

$$
\begin{gathered}
x(0)=-\frac{b}{2}, \quad x(1)=\frac{b}{2}, \quad y(0)=y(1)=0, \\
\theta(0)=\theta(1)=0 .
\end{gathered}
$$

Thus the boundary value problem for the rod model consists of $(1.8 \mathrm{a}, \mathrm{b}),(1.10)$, (1.11) along with the constituative relations (1.12) and the boundary conditions (1.17).

We can prove existence of a solution to this problem by an argument following the lines of that in [3]. This solution is found by minimizing the total energy functional over the set of all possible configurations of the rod which satisfy the boundary conditions (1.17). As we will see we would not expect this solution to be unique.

We now embed this problem in a continuum of problems by setting

$$
\Phi_{\varepsilon}(u, v)=\Phi(\varepsilon u, v)
$$

and considering the problem with stored energy function $\Phi_{\varepsilon}$ for $\varepsilon>0$. Then (1.13a) becomes

$$
\widehat{M}(u, v)=\varepsilon \Phi_{1}(\varepsilon u, v) .
$$

Here we use the notation $\Phi_{1}$ to denote the derivative of $\Phi$ with respect to its first argument, etc. Thus if $\varepsilon$ is small we see that the bending stiffness $M / u$ will be small. Formally in the limit as $\varepsilon \rightarrow 0$ we have $M \equiv 0$ and from (1.11c) $h=0$. Thus $\mathbf{n}$ is parallel to $\mathbf{r}^{\prime}$. In fact

$$
\mathbf{n}=N_{0}(v) \mathbf{e}_{1}
$$

where

$$
N_{0}(v)=\Phi_{2}(0, v)=\widehat{N}(0, v) .
$$

From (1.15a) and (1.16) we have

$$
N_{0}(1)=0, \quad N_{0}(v) \rightarrow-\infty \quad \text { as } v \rightarrow 0, \quad N_{0}(v) \rightarrow \infty \quad \text { as } v \rightarrow \infty .
$$

The convexity of $\Phi$ implies

$$
\frac{d}{d v} N_{0}(v)>0
$$

Equations (1.2), (1.20), (1.22), (1.23) describe a string theory. In the next section we will obtain solutions for the string model. In Sect. 3 we will show how to construct an asymptotic solution to the rod problem described in this section with $\Phi=\Phi_{\varepsilon}$. 
2. The string model. In this section we model the wire as a string. Thus we consider the physical situation described in Sect. 1 but now we consider the wire to be perfectly flexible. Thus $\mathbf{m}=\mathbf{0}$ and $\mathbf{n}$ satisfies (1.20). The tension $N_{0}(v)$ satisfies (1.22) and (1.23). In addition we assume

$$
N_{0}(v) / v \text { is unbounded as } v \rightarrow \infty .
$$

Since now $h \equiv 0,(1.11 \mathrm{a})$ implies that $N_{0}$ and hence $v$ is constant. Now (1.11b) becomes

$$
\theta^{\prime}=\frac{\lambda v}{N_{0}(v)}
$$

The boundary conditions are $(1.17 \mathrm{a})$. In this model we cannot impose a boundary condition on $\theta$ as this would make the problem overdetermined. This is an important point. It is this "loss of boundary condition" which gives rise to the boundary layer correction terms in the asymptotic solution constructed in Sect. 3.

Thus from $(2.1)$

$$
\theta=\theta_{0}+\frac{\lambda v}{N_{0}(v)} s
$$

where the constants $v$ and $\theta_{0}$ must be determined. We expect the solution to be symmetric with respect to $s=\frac{1}{2}$ with $x\left(\frac{1}{2}\right)=\theta\left(\frac{1}{2}\right)=0$. Hence from $(18 \mathrm{a}, \mathrm{b})$

$$
\begin{aligned}
& x=\frac{N_{0}(v)}{\lambda} \sin \frac{\lambda v}{N_{0}(v)}\left(s-\frac{1}{2}\right), \\
& y=\frac{N_{0}(v)}{\lambda} \cos \frac{\lambda v}{N_{0}(v)}\left(s-\frac{1}{2}\right)+y_{0} .
\end{aligned}
$$

The boundary conditions for $y$ will be satisfied if

$$
y_{0}=\frac{N_{0}(v)}{\lambda} \cos \frac{\lambda v}{2 N_{0}(v)}
$$

while the boundary conditions for $x$ will be satisfied if

$$
b=\frac{2 N_{0}(v)}{\lambda} \sin \frac{\lambda v}{2 N_{0}(v)} .
$$

We must use (2.6) to determine the value of the constant $v$. To see that (2.6) has (possibly many) solutions we rewrite $(2.6)$ as

$$
\frac{b}{v}=\frac{2 N_{0}(v)}{\lambda v} \sin \frac{\lambda v}{2 N_{0}(v)} \equiv \frac{\sin \alpha}{\alpha}
$$

To see that (2.7) has at least one solution for $v \in(b, \infty)$ we note that in view of (2.1)

$$
\limsup _{v \rightarrow \infty} \frac{\sin \alpha}{\alpha}=1 \text {. }
$$

This ensures that the graphs of the functions $b / v$ and $\sin \alpha / \alpha$ cross at least once for $v \in(b, \infty)$. Depending on the value of $\lambda$ and the growth of the function $N_{0}(v) / v$ there may be multiple solutions (see [4]). 
Thus we see that the string model admits solutions in which the wire assumes the shape of a circular arc and the tension is constant. In the next section we will show how any one of these solutions forms the basis for a singular perturbation scheme which is used to compute approximate solutions for the rod model with small bending stiffness.

3. The asymptotic solution. In this section we consider the rod model which consists of the Eqs. (1.8a, b), (1.10), (1.11), constituative relations (1.12) with stored energy function $\Phi_{\varepsilon}$ given by (1.18) and boundary conditions (1.17). Using the notation $\mathscr{M}=\Phi_{1}, \mathscr{N}=\Phi_{2},(1.13)$ now becomes

$$
\widehat{M}(u, v)=\varepsilon \mathscr{M}(\varepsilon u, v), \quad \widehat{N}(u, v)=\mathscr{N}(\varepsilon u, v)
$$

so that (1.11) now becomes

$$
\begin{aligned}
& \mathscr{N}^{\prime}(\varepsilon u, v)-u h=0, \\
& h^{\prime}+u \mathcal{N}(\varepsilon u, v)-\lambda v=0, \\
& \varepsilon \mathscr{M}^{\prime}(\varepsilon u, v)+v h=0 .
\end{aligned}
$$

The Eqs. (1.10) and (1.11) which we repeat here for convenience remain

$$
\begin{aligned}
& x^{\prime}=v \cos \theta, \\
& y^{\prime}=v \sin \theta, \\
& \theta^{\prime}=u .
\end{aligned}
$$

We seek an interior solution as an asymptotic series in $\varepsilon$

$$
x(s, \varepsilon)=x_{0}(s)+\varepsilon x_{1}(s)+\ldots
$$

with similar expansions for the other five variables. If we substitute these expansions into (3.2) and let $\varepsilon \rightarrow 0$ we find that the zero-order terms satisfy the string equations of Sect. 2 with

$$
N_{0}(v)=\mathscr{N}(0, v) .
$$

Condition (2.1) is a consequence of (1.14a). Let $v_{0}$ be any solution of (2.6) and

$$
N_{*}=N_{0}\left(v_{0}\right) \text {. }
$$

Then the zero-order terms are given by the solution of this string equation:

$$
\begin{aligned}
x_{0}(s)=\frac{N_{*}}{\lambda} \sin \frac{\lambda v_{0}}{N_{*}}\left(s-\frac{1}{2}\right), \\
y_{0}(s)=\frac{N_{*}}{\lambda} \cos \frac{\lambda v_{0}}{N_{*}}\left(s-\frac{1}{2}\right)-\frac{N_{*}}{\lambda} \cos \frac{\lambda v_{0}}{2 N_{*}}, \\
\theta_{0}(s)=\frac{\lambda v_{0}}{N_{*}}\left(s-\frac{1}{2}\right), \\
v_{0}(s)=v_{0}, \\
u_{0}(s)=\frac{\lambda v_{0}}{N_{*}} \equiv u_{0},
\end{aligned}
$$




$$
h_{0}(s)=0 \text {. }
$$

This solution satisfies the boundary condition (1.17a) but not (1.17b). Thus in order to obtain an asymptotic solution to our boundary value problem we must add on boundary layer correction terms. Thus we make the following ansatz: (cf. [2]) If $z$ is any component of the solution

$$
z(s, \varepsilon)=z_{0}(s)+Z(\sigma, \varepsilon)+\underline{Z}(\tau, \varepsilon)+O(\varepsilon)
$$

where the initial layer correction $Z(\sigma, \varepsilon)$ decays to zero as the stretched variable

$$
\sigma=\frac{s}{\varepsilon}
$$

tends to infinity and the terminal layer correction $\underline{Z}(t, \varepsilon)$ decays to zero as

$$
\tau=\frac{1-s}{\varepsilon}
$$

becomes infinite. Actually in our case, once we determine $Z$ we can find $\underline{Z}$ by symmetry. We will expand $Z$ in powers of $\varepsilon$. To determine the form this expansion should take for the various components we recall that the reason for introducing the boundary layer corrections is that the inner solution (3.6) does not satisfy the boundary condition $(1.17 \mathrm{~b})$ viz. $\theta_{0}(0)=0$. Thus the initial layer term must connect the value $\theta_{0}(0)=-\lambda v_{0} / 2 N_{*}$ with zero. This means that $\theta$ must change rapidly in the initial layer. Hence its derivative $u$ must be large (i.e. $O(1 / \varepsilon)$ ) there. Thus in the initial layer we assume

$$
\begin{gathered}
x(\sigma, \varepsilon)=x_{0}(\sigma \varepsilon)+X_{0}(\sigma)+\varepsilon X_{1}(\sigma)+O\left(\varepsilon^{2}\right) \\
y(\sigma, \varepsilon)=y_{0}(\sigma \varepsilon)+Y_{0}(\sigma)+\varepsilon Y_{1}(\sigma)+O\left(\varepsilon^{2}\right) \\
\theta(\sigma, \varepsilon)=\theta_{0}(\sigma \varepsilon)+\Theta_{0}(\sigma)+O(\varepsilon) \\
v(\sigma, \varepsilon)=v_{0}+V_{0}(\sigma)+O(\varepsilon) \\
u(\sigma, \varepsilon)=u_{0}+\frac{1}{\varepsilon} U_{0}(\sigma)+O(1) \\
h(\sigma, \varepsilon)=H_{0}(\sigma)+O(\varepsilon) .
\end{gathered}
$$

We will see below why we include the terms $X_{1}$ and $Y_{1}$. The initial conditions are

$$
X_{0}(0)=X_{1}(0)=Y_{0}(0)=Y_{1}(0)=0, \quad \Theta_{0}(0)=-\theta_{*} \equiv \frac{\lambda v_{0}}{2 N_{*}} .
$$

We may assume $\left|\theta_{*}\right|<\frac{\pi}{2}$ (cf. Sect. 2).

We insert (3.10) into (3.2d) and use dot to denote differentiation with respect to $\sigma$. We find

$$
x_{0}^{\prime}(\sigma \varepsilon)+\frac{1}{\varepsilon} \dot{X}_{0}(\sigma)+\dot{X}_{1}(\sigma)=\left(v_{0}+V_{0}(\sigma)\right) \cos \left(\theta_{0}(\sigma \varepsilon)+\Theta_{0}(\sigma)\right) .
$$

Letting $\varepsilon$ tend to zero we see that we must have

$$
\begin{aligned}
& X_{0}(\sigma)=0 \\
& x_{0}^{\prime}(0)+\dot{X}_{1}(\sigma)=\left(v_{0}+V_{0}(\sigma)\right) \cos \left(\theta_{*}+\Theta_{0}(\sigma)\right) .
\end{aligned}
$$


Now $x_{0}^{\prime}(0)=v_{0} \cos \theta_{*}$ so that (3.13) becomes

$$
\dot{X}_{1}(\sigma)=\left(v_{0}+V_{0}(\sigma)\right) \cos \left(\theta_{*}+\Theta_{0}(\sigma)\right)-v_{0} \cos \theta_{*} .
$$

In the same way from $(3.2 \mathrm{e})$

$$
\begin{aligned}
& Y_{0}(\sigma)=0, \\
& \dot{Y}_{1}(\sigma)=\left(v_{0}+V_{0}(\sigma)\right) \sin \left(\theta_{*}+\Theta_{0}(\sigma)\right)-v_{0} \sin \theta_{*} .
\end{aligned}
$$

Similarly $(3.2 \mathrm{f})$ yields

$$
\dot{\Theta}_{0}(\sigma)=U_{0}(\sigma) .
$$

Now inserting (3.10) into $(3.2 \mathrm{a}, \mathrm{b}, \mathrm{c})$ and letting $\varepsilon$ tend to zero we find

$$
\begin{gathered}
\dot{\mathscr{N}}\left(U_{0}(\sigma), v_{0}+V_{0}(\sigma)\right)-H_{0}(\sigma) U_{0}(\sigma)=0, \\
\mathscr{M}\left(U_{0}(\sigma), v_{0}+V_{0}(\sigma)\right)+H_{0}(\sigma)\left(v_{0}+V_{0}(\sigma)\right)=0, \\
\dot{H}_{0}(\sigma)+\mathscr{N}\left(U_{0}(\sigma), v_{0}+V_{0}(\sigma)\right) U_{0}(\sigma)=0 .
\end{gathered}
$$

Since the correction terms must tend to zero as $\sigma$ tends to infinity we require that

$$
\Theta_{0}(\sigma), U_{0}(\sigma), V_{0}(\sigma), H_{0}(\sigma) \rightarrow 0 \quad \text { as } \sigma \rightarrow \infty .
$$

We now analyze (3.18). We set

$$
W_{0}(\sigma)=v_{0}+V_{0}(\sigma)
$$

and drop the subscripts. We then rewrite (3.18) as

$$
\begin{aligned}
\dot{N}-H U & =0, \\
\dot{\mathscr{M}}+H W & =0, \\
\dot{H}+U \mathscr{N} & =0 .
\end{aligned}
$$

In view of (3.19), (3.20), (3.4), (3.5), and (1.16a) we have

$$
\mathscr{N} \rightarrow N_{*}, \quad \mathscr{M} \rightarrow 0 \quad \text { as } \sigma \rightarrow \infty .
$$

By using (3.17), (3.21), and (3.22) we can solve (3.21a) and (3.21c) to obtain

$$
\begin{aligned}
& \mathcal{N}=N_{*} \cos \Theta, \\
& H=-N_{*} \sin \Theta .
\end{aligned}
$$

We introduce a new variable $Z(\sigma)$ by

$$
Z(\sigma)=Z_{*}+N_{*} \int_{0}^{\sigma} W(\tau) \sin \Theta(\tau) d \tau
$$

where $Z_{*}$ is a constant to be determined. Equation (3.23b) can then be written

$$
\dot{\mathscr{M}}=N_{*} W \sin \Theta=\dot{Z}
$$

or integrating

$$
\mathscr{M}=Z
$$


where now $Z_{*}$ serves as the constant of integration. We now rewrite (3.25) and (3.23a) as

$$
\begin{aligned}
& \mathscr{M}(\dot{\Theta}, W)=Z, \\
& \mathscr{N}(\dot{\Theta}, W)=N_{*} \cos \Theta .
\end{aligned}
$$

Since the energy functional $\Phi$ is convex and coercive, Eq. (3.26) can be solved globally for $\dot{\Theta}$ and $W$ i.e., we can write

$$
\begin{aligned}
& \dot{\Theta}=\mathscr{U}\left(Z, N_{*} \cos \Theta\right), \\
& W=\mathscr{V}\left(Z, N_{*} \cos \Theta\right) .
\end{aligned}
$$

We multiply (3.27b) by $N_{*} \sin \Theta$ and use (3.24). Equation (3.27) then becomes

$$
\begin{gathered}
\dot{\Theta}=\mathscr{U}\left(Z, N_{*} \cos \Theta\right), \\
\dot{Z}=N_{*} \sin \Theta \mathscr{V}\left(Z, N_{*} \cos \Theta\right) .
\end{gathered}
$$

Equation (3.28) represents a two-dimensional autonomous system in the variables $\Theta$ and $Z$. The origin is a stationary point for this system. In order to understand the nature of this stationary point we linearize $(3.28)$ about $(0,0)$. We have

$$
\begin{aligned}
& \mathscr{U}_{Z}\left(0, N_{*}\right) \equiv u_{*}>0, \\
& \mathscr{V}\left(0, N_{*}\right)=v_{0},
\end{aligned}
$$

so the linearized system is

$$
\begin{aligned}
& \dot{\Theta}=u_{*} Z, \\
& \dot{Z}=N_{*} v_{0} \Theta .
\end{aligned}
$$

Clearly the system (3.30) has one positive and one negative eigenvalue. Thus there is exactly one pair of orbits of (3.28) entering the origin as $\sigma \rightarrow \infty$. Along these orbits $\Theta$ and $Z$ decay exponentially. We wish to show that for some $Z_{*}$ (cf. (3.24)) the point $\left(Z_{*},-\theta_{*}\right)$ lies on one of these orbits. It will then follow that the solution of (3.28) starting at this point at $\sigma=0$ will decay exponentially as $\sigma \rightarrow \infty$. Then from (3.23b) $H$ will decay exponentially and from (3.27b) $V=W-v_{0}$ also will decay exponentially. Finally $X_{1}$ and $Y_{1}$ can be found by integrating (3.14) and (3.16) using the initial values (3.11). These functions will have finite, but not necessarily zero, limits as $\sigma \rightarrow \infty$. However this is acceptable since, it will be recalled, these functions are multiplied by $\varepsilon$ in the expansion (3.10). Thus all the conditions of the boundary layer problem will be satisfied.

In order to analyze (3.28) we recall that the functions $\mathscr{U}$ and $\mathscr{V}$ arise from inverting the equations

$$
\begin{aligned}
& m=\mathscr{M}(u, v), \\
& n=\mathscr{N}(u, v),
\end{aligned}
$$

where $\mathscr{M}=\Phi_{u}$ and $\mathscr{N}=\Phi_{v}$. Thus if we set

$$
\Psi^{*}(m, n)=m \mathscr{U}(m, n)+n \mathscr{V}(m, n)-\Phi(\mathscr{U}(m, n), \mathscr{V}(m, n))
$$


(the Legendre-Fenchel transformation) we have

$$
\Psi_{m}^{*}=\mathscr{U}, \quad \Psi_{n}^{*}=\mathscr{V} .
$$

The function $\Psi^{*}$ is convex and coercive in $m$, i.e., $\Psi^{*}(m, n) / m \rightarrow \infty$ as $|m| \rightarrow \infty$ uniformly for $n$ in a bounded interval. Thus we see that if we set

$$
\Psi(Z, \Theta)=\Psi^{*}\left(Z, N_{*} \cos \Theta\right)
$$

then (3.28) can be written

$$
\dot{\Theta}=\Psi_{Z}, \quad \dot{Z}=-\Psi_{\Theta},
$$

i.e., the system is Hamiltonian.

Of course $\Psi$ is conserved along the orbits. What we must show is that given a value $\theta_{*}$ with $\left|\theta_{*}\right|<\frac{\pi}{2}$ we can find a $Z_{*}$ such that the orbit starting at $\left(Z_{*},-\theta_{*}\right)$ at $\sigma=0$ approaches $(0,0)$ as $\sigma \rightarrow \infty$. The orbit is identified by the condition that along the orbit

$$
\Psi(Z, \Theta)=\Psi(0,0)=\Psi^{*}\left(0, N_{*}\right) .
$$

Here we assume that $\theta_{*}<0$. We will show that the orbit entering the origin from $\Theta>0$, if traced backward, approaches the stationary point at $(0,2 \pi)$ as $\sigma \rightarrow-\infty$. This will prove the result. We show this by ruling out all other possibilities. We first observe that since $\Psi^{*}$ is coercive in $Z, Z$ must stay bounded along the orbit. Furthermore the orbit cannot cross any line $\Theta=$ constant more than twice and cannot cross the lines $\Theta=0$ or $\Theta=2 \pi$ at all. This is because $\Psi_{Z}^{*}=\mathscr{U}$ which is positive for $Z>0$ and negative for $Z<0$. Thus the orbit, on which $\Psi^{*}$ is constant, cannot cross the line $\Theta=$ constant twice on either side of the $\Theta$ axis and cannot cross $\Theta=0$ or $\Theta=2 \pi$ at all. Thus by the Poincaré-Bendixson theorem the orbit must approach a stationary point as $\sigma \rightarrow-\infty$. But the only stationary points in the strip $0 \leq \Theta \leq 2 \pi$ are at $(0,2 \pi),(0, \pi)$, and $(0,0)$. The orbit cannot approach $(0, \pi)$ because $\Psi^{*}\left(0, N_{*}\right) \neq \Psi^{*}\left(0,-N_{*}\right)$. It cannot approach $(0,0)$ because to do so it would have to cross the $\Theta$ axis at a point $\left(0, \Theta_{1}\right)$ with $\cos \Theta_{1}<1$. But again $\Psi^{*}\left(0, N_{*}\right) \neq \Psi^{*}\left(0, N_{*} \cos \Theta_{1}\right)$. The only remaining possibility is that the orbit approaches $(0,2 \pi)$ as $\sigma \rightarrow-\infty$. This completes the construction of the boundary correction terms at $s=0$.

We now consider the boundary correction terms at $s=1$. We repeat the above procedure with $\sigma=\frac{s}{\varepsilon}$ replaced by $\tau=\frac{(1-s)}{\varepsilon}$. We start with the analogue of $(3.10)$, i.e.,

$$
X(\tau, \varepsilon)=x_{0}(1-\tau \varepsilon)+\underline{X}_{0}(\tau)+\varepsilon \underline{X}_{1}(\tau)+O\left(\varepsilon^{2}\right),
$$

etc. We find $\underline{X}_{0}=\underline{Y}_{0}=0$ and derive a system analogous to (3.14), (3.16), (3.17), (3.18) with initial conditions

$$
\underline{X}_{1}(0)=\underline{Y}_{1}(0)=0, \quad \underline{\Theta}(0)=\theta_{*} \equiv-v_{0} \lambda / 2 N_{*} .
$$

The only difference between the old and new system is that there is a minus sign in front of all the derivative terms and $\theta *$ is replaced by $-\theta *$. 
It follows that the solution of the new system is

$$
\begin{aligned}
& \underline{X}_{1}(\tau)=-X_{1}(\tau), \\
& \underline{Y}_{1}(\tau)=Y_{1}(\tau), \\
& \underline{\Theta}_{0}(\tau)=-\Theta_{0}(\tau), \\
& \underline{U}_{0}(\tau)=U_{0}(\tau), \\
& \underline{V}_{0}(\tau)=V_{0}(\tau), \\
& \underline{H}_{0}(\tau)=-H_{0}(\tau),
\end{aligned}
$$

Finally we consider the first correction terms in the interior solution, the terms $x_{1}$, $y_{1}$, etc. (c.f. (3.3)). The equations for these are obtained by collecting the $O(\varepsilon)$ terms in (3.2). The result is

$$
\begin{gathered}
\mathscr{N}_{v}\left(0, v_{0}\right) v_{1}^{\prime}-u_{0} H_{1}=0, \\
H_{1}^{\prime}+N_{*} u_{1}+\mathscr{N}_{v}\left(0, v_{0}\right) u_{0} v_{1}-\lambda v_{1}=0, \\
H_{1} v_{0}=0, \\
x_{1}^{\prime}=v_{1} \cos \theta_{0}(s)-\theta_{1} v_{0} \sin \theta_{0}(s), \\
y_{1}^{\prime}=v_{1} \sin \theta_{0}(s)+\theta_{1} v_{0} \cos \theta_{0}(s), \\
\theta_{1}^{\prime}=u_{1} .
\end{gathered}
$$

The boundary conditions are

$$
x_{1}(0)=x_{1}(1)=y_{1}(0)=y_{1}(1)=0 .
$$

We will show that the only solution of (3.38), (3.39) is the trivial one. To see this, first observe that $(3.38 \mathrm{a})$ and $(3.38 \mathrm{c})$ imply that $H_{1}=0$ and $v_{1}$ is constant. We can solve $(3.38 \mathrm{~b})$ for $u_{1}$ in terms of $v_{1}$ :

$$
u_{1}=\frac{\lambda-\mathscr{N}_{v}\left(0, v_{0}\right) u_{0}}{N_{*}} v_{1} \equiv A v_{1}
$$

We observe from (3.5) that

$$
\begin{aligned}
& \int_{0}^{1} \sin \theta_{0}(s) d s=0, \\
& \int_{0}^{1} \cos \theta_{0}(s) d s=\frac{b}{v_{0}} .
\end{aligned}
$$

From (3.38f) $\theta_{1}=A v_{1} s+\theta_{1}(0)$. We integrate (3.38e) from 0 to 1 , integrate the second term on the right by parts and use $(3.41 \mathrm{a})$. We obtain

$$
0=\int_{0}^{1} \theta_{1}(s) \cos \theta_{0}(s) d s=\frac{1}{u_{0}} \sin \theta_{*}\left\{\theta_{1}(1)+\theta_{1}(0)\right\}
$$

from which it follows that

$$
A v_{1}+2 \theta_{1}(0)=0 \text {. }
$$

Next we integrate (3.38d) from 0 to 1 . The result is

$$
0=v_{1}\left\{\frac{b}{v_{0}}-\frac{b A}{u_{0}}+\frac{v_{0}}{u_{0}} A \cos \theta_{*}\right\} \text {. }
$$


From (3.40) and (3.6e)

$$
\frac{b}{v_{0}}-\frac{b A}{u_{0}}=b \frac{\mathcal{N}_{v}\left(0, v_{0}\right)}{N_{*}}, \quad \frac{v_{0}}{u_{0}} A=1-v_{0} \frac{\mathcal{N}_{v}\left(0, v_{0}\right)}{N_{*}} .
$$

Thus (3.43) becomes

$$
0=v_{1}\left\{b \frac{\mathscr{N}_{v}\left(0, v_{0}\right)}{N_{*}}+\left(1-v_{0} \frac{\mathscr{N}_{v}\left(0, v_{0}\right)}{N_{*}}\right) \cos \theta_{*}\right\} .
$$

We claim that the term in the brackets is positive. This will imply that $v_{1}=0$. Then by (3.42) $\theta_{1}(0)=0$ so that $\theta_{1}=0$. By (3.40) $u_{1}=0$ and by $(3.38 \mathrm{~d})$ and $(3.38 \mathrm{e})$ $x_{1}=y_{1}=0$ so the only solution of $(3.38)$ is the trivial one. To verify our claim we write the term in brackets as

$$
\left(b-v_{0} \cos \theta_{*}\right) \frac{\mathcal{N}_{v}\left(0, v_{0}\right)}{N_{*}}+\cos \theta_{*} .
$$

But $\cos \theta_{*}>0$ and $\cos \theta_{0}(s) \geq \cos \theta_{*}$ for $0 \leq s \leq 1$ so that

$$
\cos \theta_{*}<\int_{0}^{1} \cos \theta_{0}(s) d s=\frac{b}{v_{0}} .
$$

Therefore $b-v_{0} \cos \theta_{*}>0$ and we are done.

Our final result is as follows:

$$
x(s, \varepsilon)=x_{0}(s)+\varepsilon X_{1}\left(\frac{s}{\varepsilon}\right)-\varepsilon X_{1}\left(\frac{1-s}{\varepsilon}\right)+O\left(\varepsilon^{2}\right)
$$

where $x_{0}$ is given by $(3.6 \mathrm{a})$ and

$$
\begin{gathered}
X_{1}(\sigma)=\int_{0}^{\sigma}\left\{\left(v_{0}+V_{0}(\rho)\right) \cos \left(\theta_{*}+\Theta_{0}(\rho)\right)-v_{0} \cos \theta_{*}\right\} d \rho, \\
y(s, \varepsilon)=y_{0}(s)+\varepsilon Y_{1}\left(\frac{s}{\varepsilon}\right)+\varepsilon Y_{1}\left(\frac{1-s}{\varepsilon}\right)+O\left(\varepsilon^{2}\right),
\end{gathered}
$$

where $y_{0}$ is given by $(3.6 \mathrm{~b})$ and

$$
\begin{gathered}
Y_{1}(\sigma)=\int_{0}^{\sigma}\left\{\left(v_{0}+V_{0}(\rho)\right) \sin \left(\theta_{*}+\Theta_{0}(\rho)\right)-v_{0} \sin \theta_{*}\right\} d \rho, \\
\theta(s, \varepsilon)=\theta_{0}(s)+\Theta_{0}\left(\frac{s}{\varepsilon}\right)-\Theta_{0}\left(\frac{1-s}{\varepsilon}\right)+O(\varepsilon),
\end{gathered}
$$

where $\theta_{0}$ is given by $(3.6 \mathrm{c})$.

$$
u(s, \varepsilon)=u_{0}+\frac{1}{\varepsilon} U_{0}\left(\frac{s}{\varepsilon}\right)+\frac{1}{\varepsilon} U_{0}\left(\frac{1-s}{\varepsilon}\right)+U_{1}\left(\frac{1}{\varepsilon}\right)+\underline{U}_{1}\left(\frac{1-s}{\varepsilon}\right)+O(\varepsilon),
$$

where $u_{0}$ is given by (3.6d), $U_{0}$ by (3.17) and $U_{1}$ and $\underline{U}_{1}$ are further correction terms.

$$
v(s, \varepsilon)=v_{0}+V_{0}\left(\frac{s}{\varepsilon}\right)+V_{0}\left(\frac{1-s}{\varepsilon}\right)+O(\varepsilon)
$$

where $v_{0}$ is given by $(3.6 \mathrm{e})$.

$$
h(s, \varepsilon)=H_{0}\left(\frac{s}{\varepsilon}\right)-H_{0}\left(\frac{1-s}{\varepsilon}\right)+O(\varepsilon) .
$$


In (3.44) the functions $V_{0}, \Theta_{0}$, and $H_{0}$ are determined as follows: First, $\left(Z, \Theta_{0}\right)$ is the solution (3.34) constructed above. Then $W_{0}$ can be found from (3.27b). Finally, $V_{0}$ is found from (3.20) and $H_{0}$ from (3.23b). Also, as we have shown, the $O(\varepsilon)$ terms in $(3.44 \mathrm{e}, \mathrm{f}, \mathrm{g}, \mathrm{h})$ involve only boundary layer terms, as we have seen that there are no $O(\varepsilon)$ terms in the interior solution.

In principle we could compute higher order correction terms both in the interior and in the boundary layers. Of course to do this we would have to assume additional smoothness of $\Phi$.

\section{REFERENCES}

[1] S. S. Antman and J. E. Dunn, Qualitative behavior of buckled nonlinear elastic arches, J. Elasticity 10, 225-239 (1980)

[2] J. E. Flaherty and R. E. O'Malley, Singularly perturbed boundary value problems for nonlinear systems, including a challenging problem for a nonlinear beam, Theory and Applications of Singular Perturbations (W. Eckhaus and E. M. deJager, eds.), Lecture Notes in Math., no. 942, Springer, Berlin-New York, 1982, pp. 170-191

[3] T. Seidman and P. Wolfe, Equilibrium states of an elastic conducting rod in a magnetic field, Arch. Rational Mech. Anal. 102, 307-329 (1988)

[4] P. Wolfe, Bifurcation theory of an elastic conducting wire subject to magnetic forces, J. Elasticity (to appear). 